\title{
miR-934 as a Prognostic Marker Facilitates Cell Proliferation and Migration of Pancreatic Tumor by Targeting PROXI
}

This article was published in the following Dove Press journal: OncoTargets and Therapy

\author{
Yangbing Jin (1) $1,2, *$ \\ Yuanchi Weng ${ }^{1,2, *}$ \\ Yue Wang ${ }^{1,2, *}$ \\ Jiewei Lin ${ }^{1,2}$ \\ Xiaxing Deng ${ }^{1,2}$ \\ Baiyong Shen ${ }^{1,2}$ \\ Qian Zhan ${ }^{1,2}$ \\ Xiongxiong $\mathrm{Lu}^{\mathrm{I}, 2}$
}

'Pancreatic Disease Center, Ruijin Hospital, Shanghai Jiaotong University School of Medicine, Shanghai 200025, People's Republic of China; ${ }^{2}$ Research Institute of Pancreatic Disease, Shanghai Jiaotong University School of Medicine, Shanghai 200025, People's Republic of China

*These authors contributed equally to this work
Correspondence: Qian Zhan; Xiongxiong Lu

Pancreatic Disease Center, Ruijin Hospital, Shanghai Jiaotong University School of Medicine, 197 Ruilin 2 Road, Shanghai 200025, People's Republic of China

Email zhanxi80@।26.com;

simone5I5night@I26.com
Background: Pancreatic cancer is an extremely lethal digestive cancer with late diagnosis and poor prognosis. miR-934 has been reported to serve as an oncogene in multiple cancers, such as ovarian cancer and bladder cancer. However, its role in pancreatic cancer remains undiscovered.

Materials and Methods: The expression data of miR-934 were obtained from the Gene Expression Omnibus database and from our own patient samples. The clinicopathological data and corresponding follow-up data were retrieved from The Cancer Genome Atlas database. CCK8 and colony formation assays were conducted to measure cell proliferation capacity in vitro. Wound healing and transwell assays were performed to detect the migration ability of pancreatic cancer cell.

Results: We found that miR-934 was significantly upregulated in pancreatic tumor samples and cell lines. The expression of miR-934 was related to pathological stages. Upregulated miR-934 was associated with poor prognosis in patients with pancreatic cancer. Mir-934 inhibition reduced, while overexpression promoted, cell proliferation and migration. Mechanically, we found miR-934 could directly bind to 3'-UTR of PROX1 leading to mRNA derogation. Furthermore, increased cell proliferation and migration caused by miR934 overexpression could be reversed by forced PROX1 expression.

Conclusion: miR-934 is an oncogene in pancreatic cancer and could serve as a prognosis indicator for patients with pancreatic cancer, suggesting that miR-934 is a promising therapeutic target for pancreatic cancer.

Keywords: miR-934, PROX1, proliferation, metastasis, pancreatic cancer

\section{Introduction}

Pancreatic cancer is one of the most malignant tumors of the digestive system, with extremely poor prognosis. The 5-year survival rate is less than $5 \%$. More than $90 \%$ of pancreatic cancer is pathologically diagnosed as pancreatic ductal adenocarcinoma (PDAC). Although significant progress in surgery and medical treatment has been made in the past several years, the overall efficacy still remains unsatisfactory. ${ }^{1}$ Unclear causes, low early-diagnosis rate, and obscure mechanism of tumor development are currently the main challenges for the treatment of pancreatic cancer. ${ }^{2}$ In the new era of precision medicine, it is urgent to develop early diagnosis and individualized treatment strategy at the molecular level, improving the detection rate of high-risk groups and the prognosis of patients with pancreatic cancer. 
MicroRNAs are a class of non-coding single-stranded RNA molecules of approximately 22 nucleotides in length, which play an extensive role in the gene expression network. The importance of miRNAs in tumor molecular biology has been demonstrated in multiple studies. ${ }^{3}$ Through regulating mRNA translation in a positive or negative way, miRNAs participate in various tumor biological behavior such as growth, invasion, metastasis, and angiogenesis. ${ }^{4}$ MicroRNA 934 has been reported as an oncogene in some literature. For instance, it has been found that in head and neck squamous cell cancer, treatment with ethanol and acetaldehyde leads to upregulation of miR-934, promotes tumor cell proliferation and induces overexpression of anti-apoptotic genes. ${ }^{5}$ In ovarian cancer, miR-934 served as an oncogene by directly targeting BRMS1L. ${ }^{6}$ Recent evidence also suggested that miR-934 targeting UBE2N could promote cell growth of bladder cancer by inhibiting CDK6 degradation. ${ }^{7}$

The PROX1 encoding protein is a member of the homeobox transcription factor family and its function is mostly involved in lymphangiogenesis and angiogenesis. ${ }^{8}$ Multiple studies have shown a close relationship between PROX1 and tumors, but due to the complexity of the interaction between pathways, PROX1 may play a completely paradoxical role in tumors. ${ }^{9-11}$

Previous studies have found that PROX1 plays a role in the development of the exocrine pancreas ${ }^{12}$ and the secondary functional transition of pancreatic cells. ${ }^{13}$ Moreover, Drosos and his colleges constituted that PROX1 activity can reduce anchorage-independent growth and transformation, invasion in pancreatic cancer cells. ${ }^{12}$ Saukkonen showed that PROX1, mainly expressed in the cytoplasm of PDAC cells, could be used as an independent predictor of better prognosis of pancreatic cancer. ${ }^{14}$

\section{Materials and Methods}

\section{Patients and Samples}

The clinicopathological and follow-up data of miR-934 including 148 pancreatic cancer patients were downloaded from The Cancer Genome Atlas (TCGA) database. Expression data were derived from GSE85589, which contains 16 tumor tissues and 16 adjacent normal tissues. Besides, we collected 14 pancreatic cancer samples and 12 adjacent normal tissues from patients treated in our center. All patients involved in this study had read and signed written informed consent. The research protocol had been approved by the Ethics Committee of Shanghai Ruijin
Hospital (No.17 in 2019). Related experiments involving human specimens were in strict accordance with the Ethics Committee of Human Experimentation and with the Declaration of Helsinki as revised in 2013.

\section{Cell Culture}

Four cell lines, including normal human pancreatic ductal cell line HPNE and pancreatic tumor cell lines (Capan-1, BxPC-3 and Patu-8988) were obtained from the cell bank of Chinese Academy of Sciences. The cells were cultured in RPMI 1640, DMEM supplemented with $10 \%$ fetal bovine serum (FBS) and $100 \mathrm{U} / \mathrm{mL}$ penicillin and $100 \mathrm{U} / \mathrm{mL}$ streptomycin. All cells were incubated in a humidified air with $5 \% \mathrm{CO}_{2}$ at $37^{\circ} \mathrm{C}$.

\section{Western Blot Assay}

Different types of cells were lysed using RIPA buffer (Sigma-Aldrich; R0278). The obtained protein samples were separated by sodium dodecyl sulfate-polyacrylamide gel electrophoresis (SDS-PAGE) and transferred to a polyvinylidene difluoride (PVDF) membrane. It was then blocked with $5 \%$ skim milk for $1 \mathrm{~h}$ and incubated with specific primary antibody overnight at $4{ }^{\circ} \mathrm{C}$. After incubation with an appropriate secondary antibody for 1 hour at room temperature, the target protein component separated by the electrophoresis could be detected by autoradiography.

\section{RNA Extraction and Real-Time Quantitative PCR (qRT-PCR)}

Total RNA was isolated from cells using Trizol reagent according to standard protocols provided by the manufacturer and then was reverse transcribed into cDNA using the M-MLV reverse transcriptase kit (Invitrogen). GAPDH was used as internal control. The primer sequences used for the detection of miR-934 and GAPDH are presented as follows:

\section{CCK-8 Assay}

The cells were seeded at a density of 2000 per well into 96-well plates and cultured for 24 hours. Thereafter, $110 \mu \mathrm{L}$ of the complete culture solution containing $10 \mu \mathrm{L}$ of CCK-8 reagent was added to each well every 24 hours, and the mixture was incubated in a $37^{\circ} \mathrm{C}$ incubator for 2 hours. The absorbance at $450 \mathrm{~nm}$ was measured using a microplate reader (Epoch; BioTek, Winooski, VT).

\section{Colony Formation Assay}

The cells were seeded into a 6-well plate at a density of 1000 per well. After 2 weeks of incubation, the cells were 
stained with a $0.1 \%$ crystal violet solution, and the number of colonies was counted to evaluate the degree of cell proliferation. The images were obtained at $\times 100$ magnification.

\section{Wound Healing Assay}

Cells were seeded into 6-well plates and incubated overnight, and the next day, a wound of cell monolayer was created, then cultured in serum-free medium, and cells distance was estimated using Image $\mathbf{J}$ at different time points. The images were obtained at $\times 100$ magnification.

\section{Transwell Assay}

The cells to be tested were cultured in serum-free medium for 24 hours, and then inoculated into an upper chamber containing $150 \mu \mathrm{L}$ of serum-free medium at a cell density of 50,000 per well. A complete medium containing $10 \%$ fetal calf serum was added to the lower chamber. Incubate for 24 hours at $37^{\circ} \mathrm{C}$ in a $5 \% \mathrm{CO}_{2}$ incubator and then stain with crystal violet. Five fields were randomly taken under the microscope to perform cell counting and statistical results.

\section{Lentiviral Transduction}

The transfection steps were performed according to the instructions provided by Ribobio Biotech (Shanghai, China) with riboFECT тм CP Reagent. The transfection concentration of miR-934-mimic was $50 \mathrm{nM}$, and the transfection concentration of miR-934-inhibitor was 200nM. The lentiviruses containing the miR-934 mimic and miR934-inhibitor plasmid vectors were constructed and transfected into Patu-8988 and Capan-1 cell lines, respectively. The lentiviruses containing the miR-934 mimic and miR934 plasmid vectors were separately constructed and transfected into $293 \mathrm{~T}$ cell line. In addition, the lentivirus containing the plasmid vector of the PROX1 gene was transfected into Patu-8988 cells to exogenously overexpress the PROX1 gene.

\section{Dual-Luciferase Reporter Assay}

StarBase software was used to predict the binding sites of the miR-934 and the 3'-UTR region of PROX1 mRNA. The mRNA sequence was mutated to synthesize the wild type (Wt) and mutant (Mut) 3'-UTR regions of PROX1. The 3'UTR regions of the wild-type and mutant PROX1 gene were ligated into the vector reporter gene to construct dual-luciferase and transfected into 293T NC and miR-934-mimic cell lines, respectively. The luciferase activity was measured 48 hours after the plasmid was co-transfected.

\section{Statistical Analysis}

All data were statistically analyzed and presented using SPSS20.0 and GraphPad Prism 5 software, and the data were expressed as mean \pm standard deviation. Pearson $\chi^{2}$ was used to detect the correlation between miR-934 expression levels and clinical pathology data. Overall survival analysis was assessed using the Kaplan-Meier method. All experiments were performed at least three times. A value of $\mathrm{P}<0.05$ was considered to be statistically significant.

\section{Results}

\section{The Expression Level of miR-934 Was Increased in PDAC Tissues and Cell Lines}

To clarify the expression level of miR-934 in pancreatic cancer, we used Gene Expression Omnibusthe database (GSE85589), clinical specimens and cultured cells to measure the expression of miR-934. As is shown is (Figure 1A), using expression data from GES85589, we found that miR-934 was significantly upregulated in tumor tissue compared with normal tissue. The result was further confirmed using 12 normal samples and 14 tumor samples from our center (Figure 1B). Besides, the expression level of miR-934 was increased in pancreatic cancer cell lines compared with normal pancreatic ductal cell (Figure 1C). With respect to its prognostic value, Kaplan-Meier curve analysis showed pancreatic cancer patients with high expression of miR-934 had significantly reduced overall survival (Figure 1D).

\section{Relationship Between miR-934 Expression Level and Clinicopathological Features of PDAC Patients}

To determine the association between miR-934 expression levels and clinicopathological features, we screened 139 eligible pancreatic cancer patients from The Cancer Genome Atlas (TCGA) and downloaded their miRNA expression levels and clinical data packages for analysis. Patients included in the analysis were divided into miR-934 low expression group $(n=69)$ and miR-934 high expression group $(n=70)$ according to the expression level of miR-934. (Definition of cut-off value: the median value of the miR-934 level). As shown in Table 1, high level of 


\section{A}
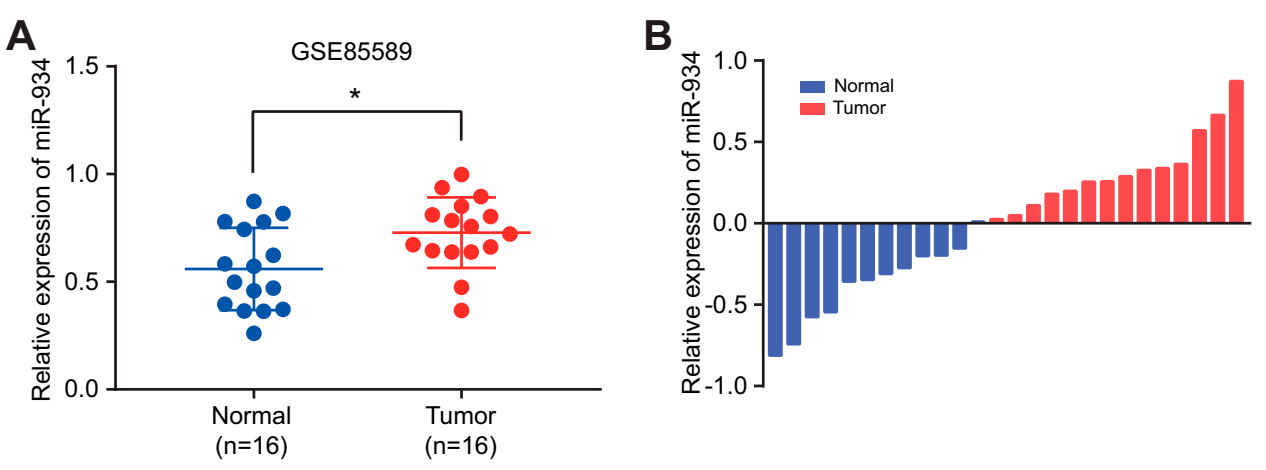

C

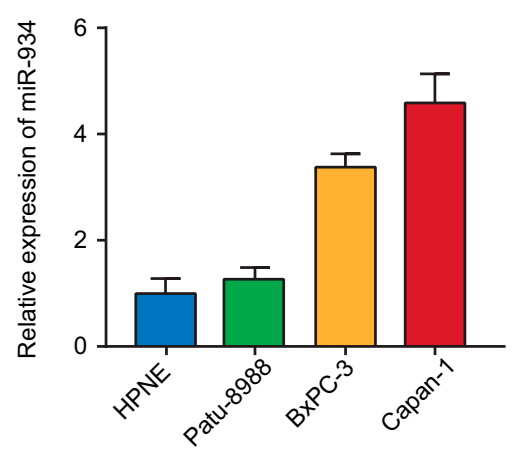

D

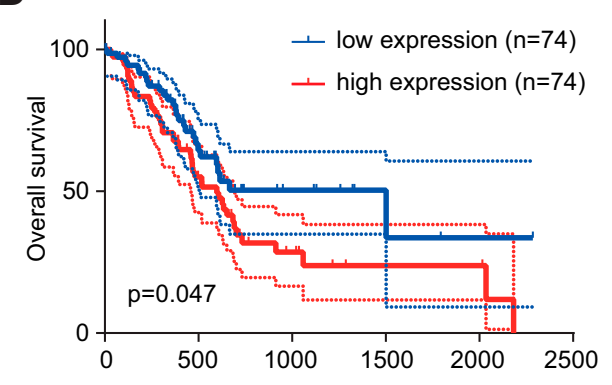

Figure I miR-934 expression and prognostic potential in pancreatic cancer. (A) Relative miR-934 expression level in GSE85589 compared I6 tumor tissues with I6 normal tissues. (B) Relative miR-934 expression using 12 normal tissues and 14 tumor tissues collected from our center. (C) Relative miR-934 expression level in normal pancreatic ductal cell line (HPNE) and three pancreatic cancer cell lines (Patu-8988, BxPC-3 and Capan-1). (D) Kaplan-Meier curves divided by miR-934 expression using survival data of patients from TCGA database. $* \mathrm{P}<0.05$ was considered statistically significant.

miR-934 expression is significantly associated with more advanced pathological stages $(\mathrm{P}=0.030)$, while age, gender, TNM stage and R stage are irrelevant.

\section{Relationship Between miR-934 Expression Level and Prognosis of PDAC Patients}

In order to determine whether miR-934 could serve as a prognostic marker, we generated the Kaplan-Meier curve using survival data of patients from the TCGA database. The results showed that patients with higher expression of miR-934 had significantly reduced overall survival rate $(\mathrm{P}=0.047$, Figure $1 \mathrm{D})$. In addition, the Cox regression model was used for univariate and multivariate analysis to identify prognostic factors associated with pancreatic cancer. As is shown in Table 2, univariate analysis showed that the $\mathrm{N}$ stage $(\mathrm{P}=0.002)$, the $\mathrm{R}$ stage $(\mathrm{P}=0.034)$ and the expression of miR-934 $(\mathrm{P}=0.015)$ were significantly associated with the prognosis of patients with pancreatic cancer, while multivariate analysis showed that the $\mathrm{N}$ stage $(\mathrm{P}=0.003)$ and the expression of miR-934 $(\mathrm{P}=0.031)$ were independent predictors of prognosis for patients with pancreatic cancer.

\section{miR-934 Enhanced the Proliferation Capacity of PDAC Cells}

To investigate the effect of miR-934 on the proliferation of pancreatic cancer cells, we established two cell models using Patu-8988 and Capan-1 that stably expressing or silencing miR-934. The transfection efficiency was examined by qRT-PCR (Figure 2A and B). CCK8 assay demonstrated that pancreatic cancer cells with increased miR-934 expression level exhibited significantly enhanced proliferation capacity (Figure 2C and D). Moreover, upregulated miR-934 could significantly promote colony formation of pancreatic cancer cell (Figure 2E and F).

\section{miR-934 Promoted the Migration Ability of PDAC Cells}

To discover the effect of miR-934 on the migration ability of pancreatic cancer cells, we performed wound healing assay. It was found that miR-934 overexpression could increase the ability of cell migration. Conversely, cells with suppressed expression of miR-934 showed reduced migration ability (Figure 3A and B). Similarly, transwell assays also showed that the migration ability of pancreatic 
Table I The Correlation Between miR-934 Expression and Clinicopathological Features of Patients with Pancreatic Cancer

\begin{tabular}{|c|c|c|c|c|c|}
\hline \multirow[t]{2}{*}{ Characteristics } & & \multirow[t]{2}{*}{$n=139$} & \multicolumn{2}{|l|}{ miR-934 } & \multirow[t]{2}{*}{$P$ value } \\
\hline & & & Low Expression & High Expression & \\
\hline Age & $\begin{array}{l}<60 \text { years } \\
\geq 60 \text { years }\end{array}$ & $\begin{array}{l}4 \mathrm{I}(29.5 \%) \\
98(70.5 \%)\end{array}$ & $\begin{array}{l}19(46.3 \%) \\
50(51.0 \%)\end{array}$ & $\begin{array}{l}22(53.7 \%) \\
48(49.0 \%)\end{array}$ & 0.615 \\
\hline Gender & $\begin{array}{l}\text { Male } \\
\text { Female }\end{array}$ & $\begin{array}{l}63(45.3 \%) \\
76(54.7 \%)\end{array}$ & $\begin{array}{l}32(50.8 \%) \\
37(48.7 \%)\end{array}$ & $\begin{array}{l}31(49.2 \%) \\
39(51.3 \%)\end{array}$ & 0.804 \\
\hline Pathologic Stage & $\begin{array}{l}\text { I+II } \\
\text { III+IV }\end{array}$ & $\begin{array}{l}|3|(94.2 \%) \\
8(5.8 \%)\end{array}$ & $\begin{array}{l}68 \text { (51.9\%) } \\
\text { I (12.5\%) }\end{array}$ & $\begin{array}{l}63(48.1 \%) \\
7(87.5 \%)\end{array}$ & 0.030 \\
\hline T Stage & $\begin{array}{l}\mathrm{TI}+\mathrm{T} 2 \\
\mathrm{~T} 3+\mathrm{T} 4\end{array}$ & $\begin{array}{l}21(15.1 \%) \\
118(84.9 \%)\end{array}$ & $\begin{array}{l}\text { II (52.4\%) } \\
58(49.2 \%)\end{array}$ & $\begin{array}{l}10(47.6 \%) \\
60(50.8 \%)\end{array}$ & 0.785 \\
\hline N Stage & $\begin{array}{l}\text { No } \\
\text { NI } \\
\text { N2 }\end{array}$ & $\begin{array}{l}37(26.6 \%) \\
59(42.4 \%) \\
43(31.0 \%)\end{array}$ & $\begin{array}{l}17(45.9 \%) \\
31 \text { (52.5\%) } \\
21 \text { (48.8\%) }\end{array}$ & $\begin{array}{l}20(54.1 \%) \\
28(47.5 \%) \\
22(51.2 \%)\end{array}$ & 0.814 \\
\hline M Stage & $\begin{array}{l}\text { MO } \\
M I \\
M x\end{array}$ & $\begin{array}{l}66(47.5 \%) \\
5(3.6 \%) \\
68(48.9 \%)\end{array}$ & $\begin{array}{l}3 \text { I (47.0\%) } \\
\text { I (20.0\%) } \\
37(54.4 \%)\end{array}$ & $\begin{array}{l}35(53.0 \%) \\
4(80.0 \%) \\
31(45.6 \%)\end{array}$ & 0.277 \\
\hline R Stage & $\begin{array}{l}\text { R0 } \\
\text { RI } \\
\text { R2 } \\
\text { Rx }\end{array}$ & $\begin{array}{l}83(59.6 \%) \\
50(36.0 \%) \\
3(2.2 \%) \\
3(2.2 \%)\end{array}$ & $\begin{array}{l}44(53.0 \%) \\
22(44.0 \%) \\
2(66.7 \%) \\
\text { I (33.3\%) }\end{array}$ & $\begin{array}{l}39(47.0 \%) \\
28(56.0 \%) \\
\text { I (33.3\%) } \\
2(66.7 \%)\end{array}$ & 0.641 \\
\hline
\end{tabular}

Table 2 Univariate and Multivariate Analyses of Clinicopathological Parameters of Patients with Pancreatic Cancer in TCGA Database by Cox-Regression

\begin{tabular}{|l|l|l|l|l|l|l|}
\hline \multirow{2}{*}{ Characteristics } & \multicolumn{2}{l|}{ Univariate Analysis } & \multicolumn{2}{l|}{ Multivariate Analysis } \\
\cline { 2 - 7 } & P value & HR & $\mathbf{9 5 \%} \mathbf{~ C l}$ & P value & HR & $\mathbf{9 5 \%}$ Cl \\
\hline N stage & 0.002 & 1.635 & $1.201-2.225$ & 0.003 & 1.603 & $1.169-2.199$ \\
R stage & 0.034 & 1.418 & $1.027-1.957$ & 0.089 & 1.336 & $0.957-1.864$ \\
Expression of miR-934 & 0.015 & 1.583 & $1.179-2.13$ & 0.031 & 1.534 & $1.152-2.785$ \\
\hline
\end{tabular}

Abbreviations: TCGA, The Cancer Genome Atlas; HR, hazard ratio; Cl, confidence interval.

cancer cells was positively related to the expression level of miR-934. After quantitative analysis, the results were statistically significant between miR-934 mimic or inhibitor group and their control groups (Figure $3 \mathrm{C}$ and $\mathrm{D}$ ).

\section{miR-934 Directly Targeted and Inhibited PROXI Expression}

To investigate the molecular mechanism by which miR934 promoted growth and metastasis of pancreatic cancer cells, we predicted the potential target of miR-934 using TargetScan. The potential targets were then subjected to correlation analysis performed by starBase 3.0 using expression data from TCGA. We found that PROX1 was a potential target and negatively associated with the expression of miR-934 (Figure 4A). To further validate the results, we performed Western blot and qRT-PCR using cells overexpressing or silencing miR-934. As is shown in Figure 4B, the protein expression level of PROX1 was negatively related to miR-934 expression level. The gray level was quantified using imageJ (Figure 4C). Similarly, the PROX1 mRNA level was also reversely associated with miR-934 expression level (Figure 4D). To discover the regulatory mechanism between miR-934 and PROX1, we obtained the potential binding target from TargetScan (Figure 4E) and performed dualluciferase reporter assay. The results showed that 293T cells transfected with wild-type PROX1 and miR-934 mimics exhibited significantly attenuated luciferase 
A

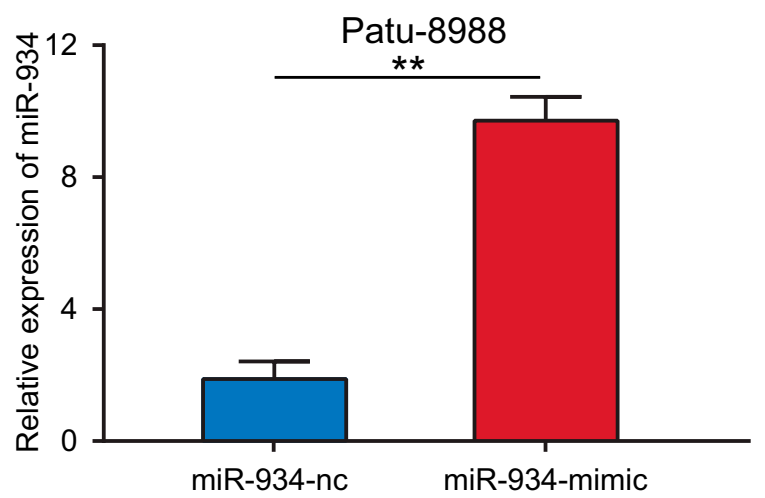

C

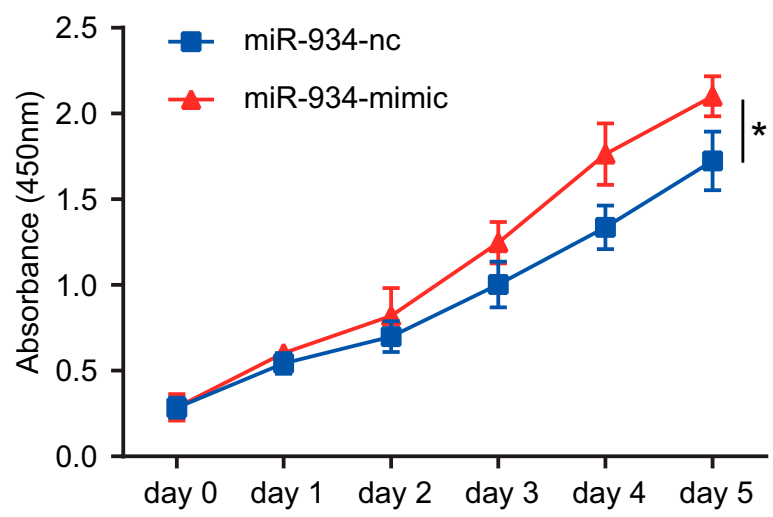

E

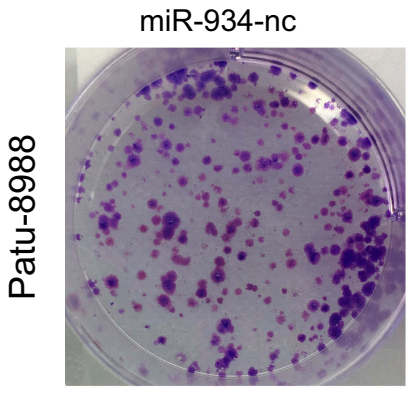

F

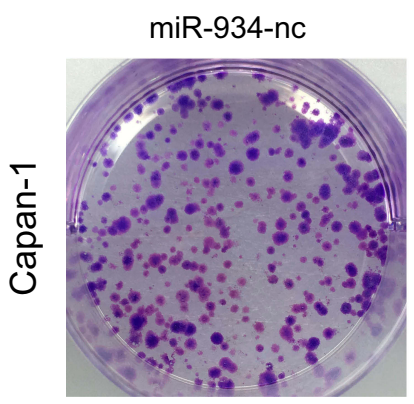

miR-934-mimic

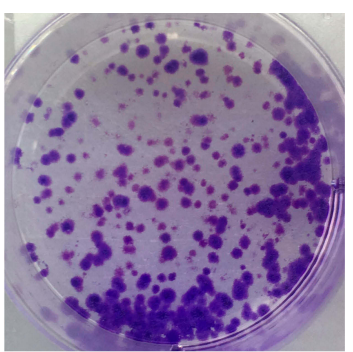

miR-934-inhibitor

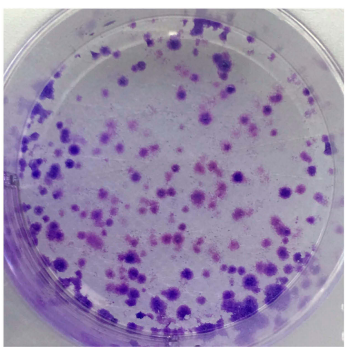

B

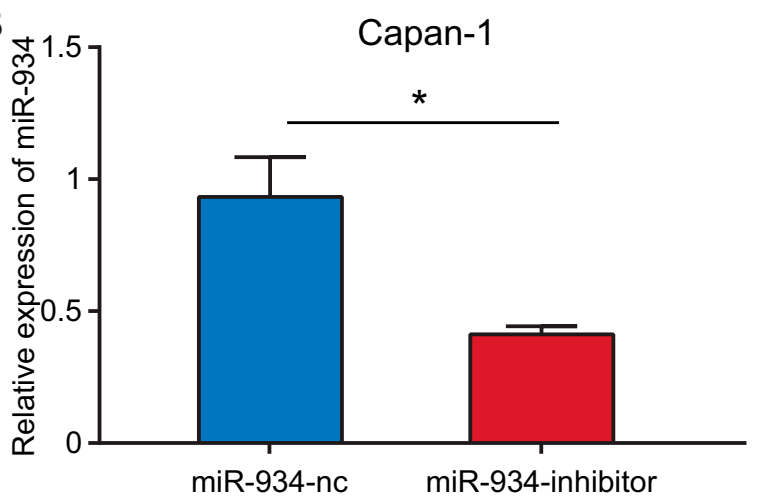

D
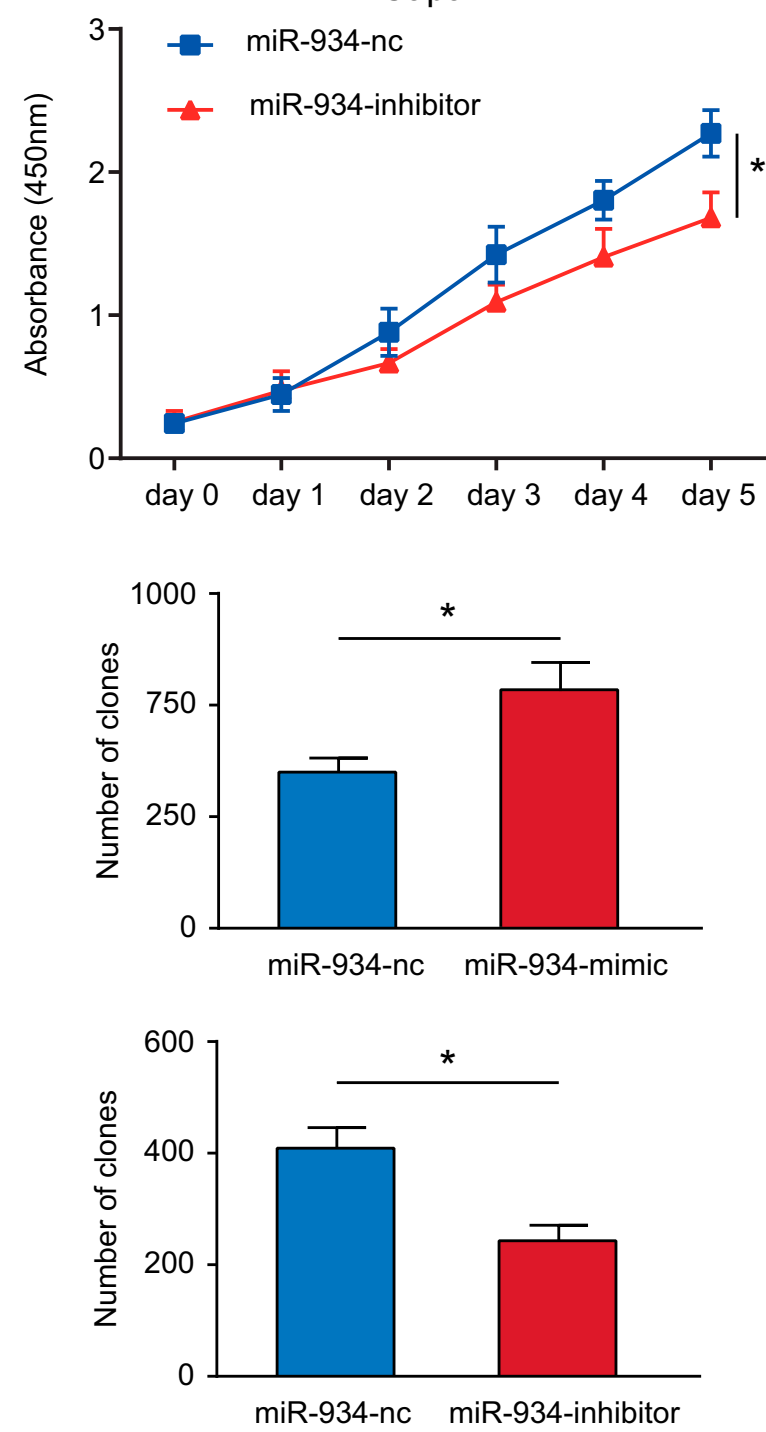

Figure 2 miR-934 enhanced cell proliferation of pancreatic cancer. (A, B) Lentiviral transfection efficiency in Patu-8988 and Capan-I cell lines determined by qRT-PCR. (C, D) The cell viability of different miR-934 expression levels was examined by CCK-8 assay. (E, F) Left panel: colony formation assay for detecting cellular proliferation capacity corresponding to different miR-934 expression levels. Right panel: the colony number was quantified by image. $* \mathrm{P}<0.05$, $* * \mathrm{P}<0.01$.

activity compared with cells with wild-type PROX1 and miR-934-nc, while transfected with mutant PROX1 vector and miR-934 mimics showed no significant change (Figure 4F). 
A
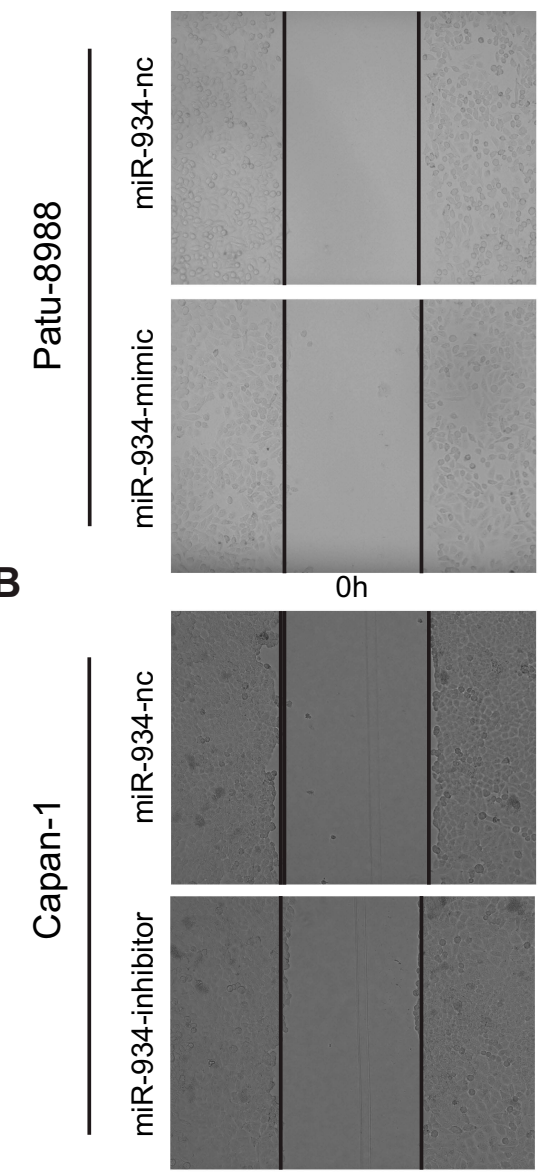

C
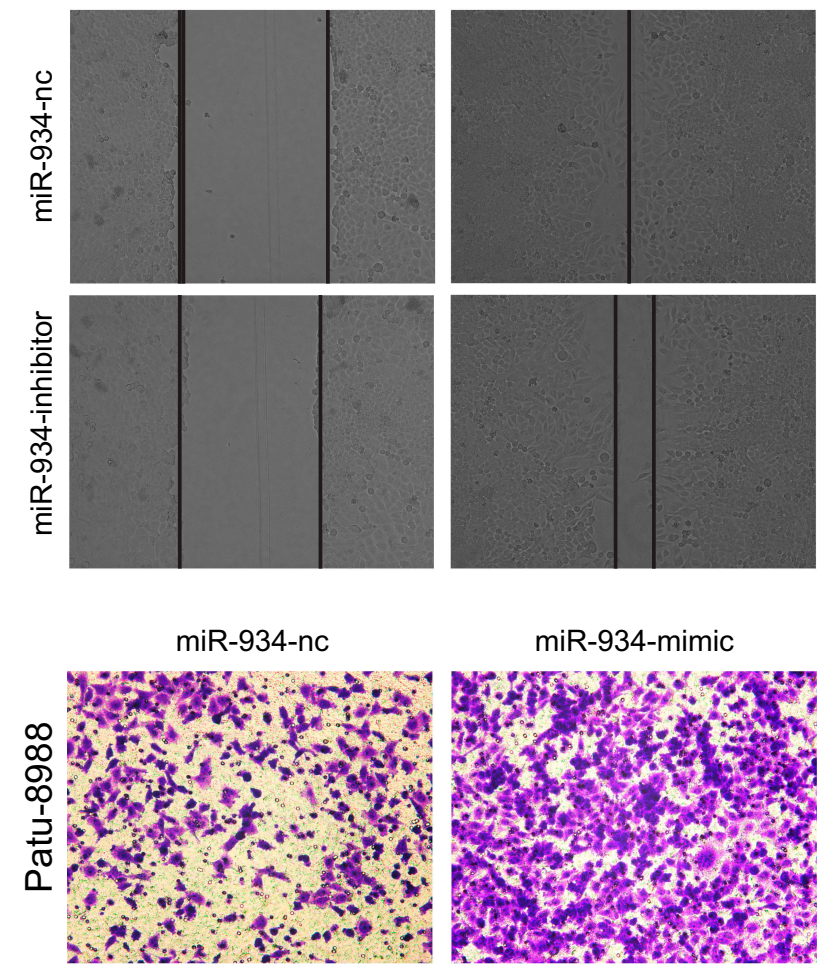

miR-934-mimic

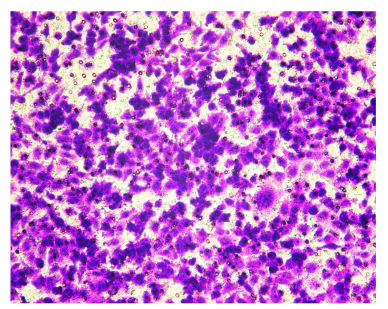

D
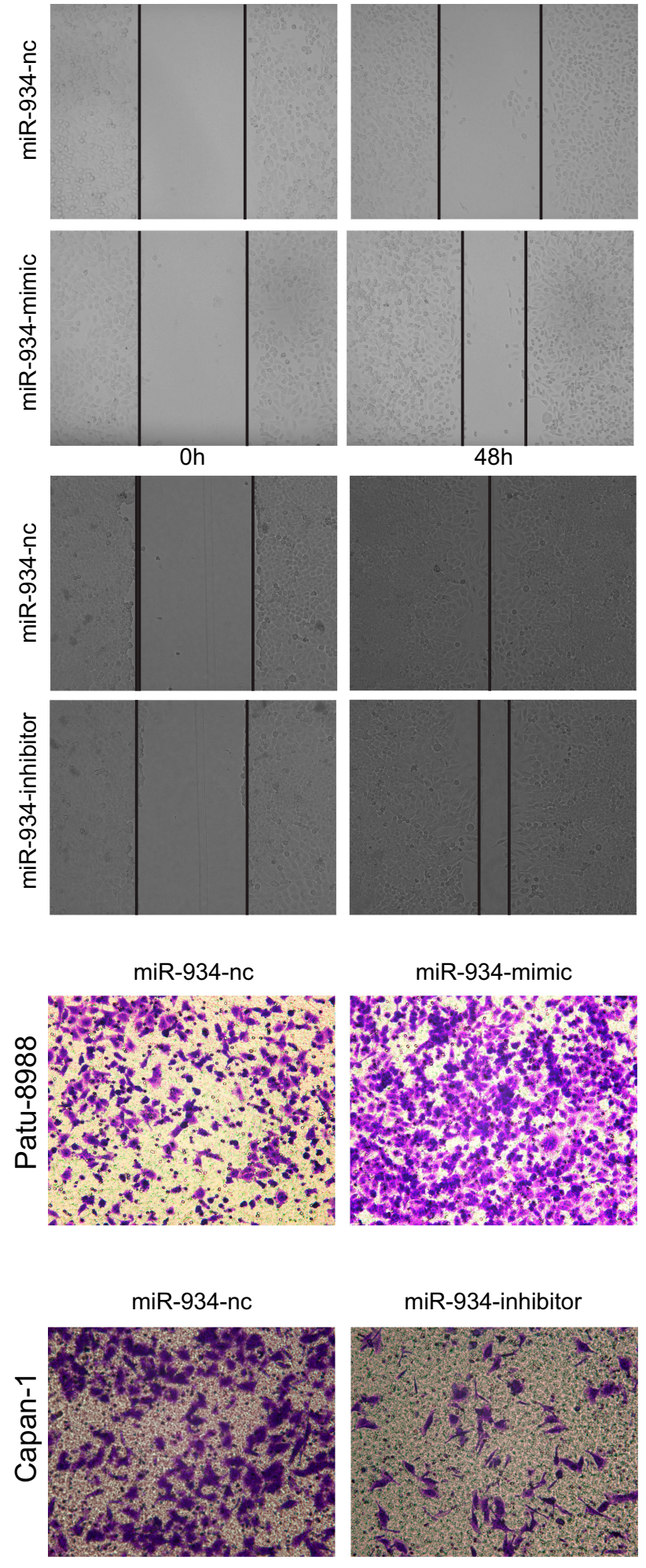

$48 \mathrm{~h}$
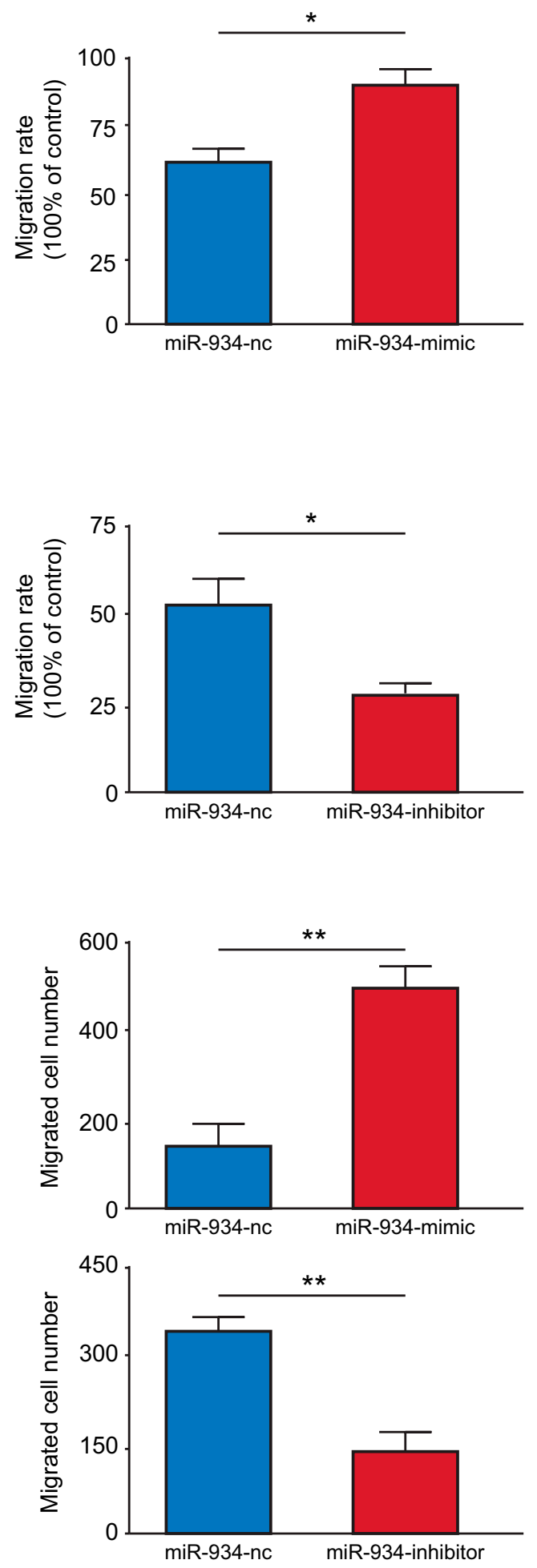

Figure 3 miR-934 promoted cell migration of pancreatic cancer. (A, B) Left panel: wound healing assay to measure the migration ability of Patu-8988 and Capan-I cells transfected with miR-934 mimic and miR-934-inhibitor, respectively. Right panel: the migration distance was quantified by imagej. (C, D) Left panel: transwell assay to detect migration ability of Patu-8988 and Capan-I cells transfected with miR-934 mimic and miR-934-inhibitor, respectively. Right panel: the migrated cells were quantified by image. $* \mathrm{P}<0.05, * * \mathrm{P}<0.01$. 
A

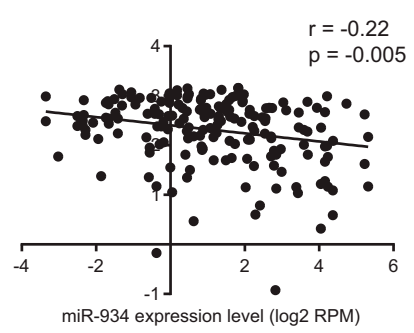

D

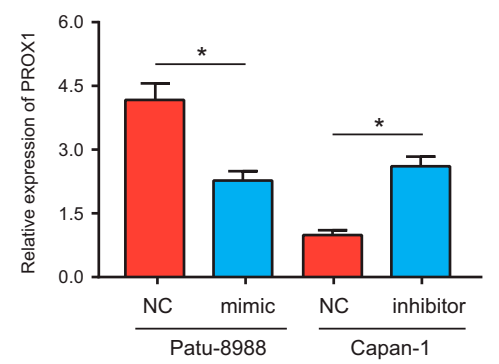

$\mathbf{B}$

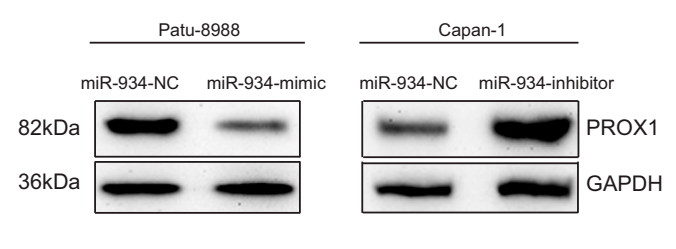

E

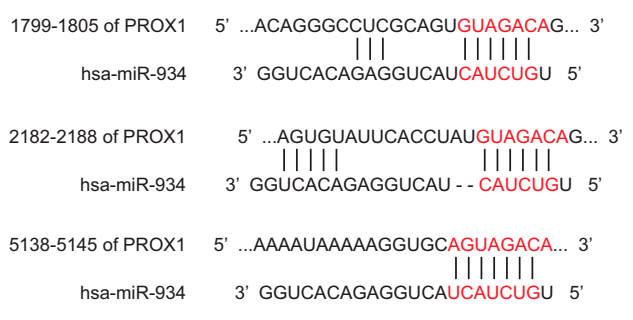

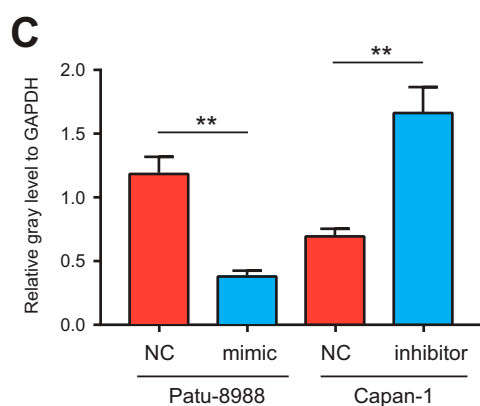

$\mathbf{F}$

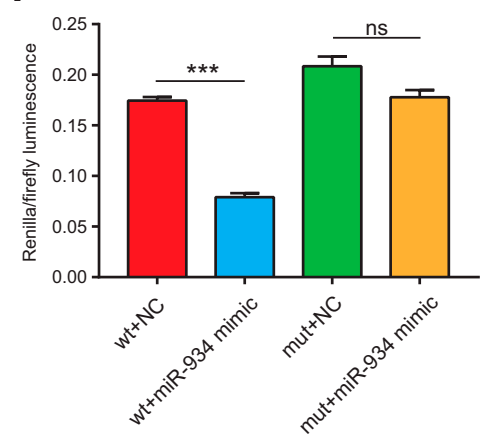

Figure 4 miR-934 directly targeted and inhibited PROXI expression. (A) The correlation analysis between miR-934 and PROXI performed using expression data from the TCGA database. (B) Western blot analysis used to detect the expression of PROXI in different miR-934 expression cell lines. GAPDH was used as an internal control. (C) The relative gray level of Western blot was quantified and analyzed. (D) PROXI mRNA expression level in cells transfected with miR-934 mimics or inhibitor was measured by qRT-PCR. (E) Potential binding targets of miR-934 to PROXI predicted by the targetScan database. (F) Dual-luciferase reporter assay used to verify the direct binding of miR-934 to PROXI. $* \mathrm{P}<0.05$, $* * \mathrm{P}<0.01$, $* * * \mathrm{P}<0.001$, ns: not significant.

\section{PROXI Is Necessary for miR-934 to Exert Its Oncogenic Function}

To verify whether miR-934 regulates cell proliferation and metastasis through PROX1, we performed rescue assay. Three cell models using Patu- 8988 were established: patu8988 transfected with miR-934-nc, patu-8988 with miR934 mimics and Patu- 8988 with miR-934 mimics and PROX1 overexpression plasmid. The transfection efficiency was detected by Western blot and qRT-PCR (Figure 5A-C). PROX1 re-overexpression could reverse the enhanced cell proliferation and migration induced by miR-934 overexpression measured by CCK 8 and colony formation assay (Figure 5D and E). Similarly, the wound healing and transwell assays showed that increased cell migration caused by miR-934 was also reversed by upregulated PROX1 (Figure 5F and G).

\section{Discussion}

MicroRNA research in pancreatic cancer has been involved in all aspects, and its importance has been self-evident. ${ }^{15}$ For example, Gregory and his colleagues found that all members of the microRNA-200 family (miR-200a, miR-200b, miR200c, miR-141 and miR-429) and miR-205 significantly down-regulated TGF- $\beta$ or ectopic expression of the protein tyrosine phosphatase Pez in cells undergoing EMT transformation. Mandatory expression of only the miR-200 family is sufficient to prevent TGF- $\beta$ induced EMT. ${ }^{16}$ Moreover, microRNA is also a novel tumor marker in clinical applications. In pancreatic cancer, several studies have demonstrated its role in diagnosis, prognosis, and as a potential therapeutic target. ${ }^{17}$ Besides, due to its relative stability of miRNAs and easy extraction of miRNAs in body fluids such as plasma, it has become a novel biomarker for non-invasive detection of cancer. ${ }^{18}$ Although there are few studies on miR-934 in the field of tumors, it has been proved to play a role in the development of various tumors. In addition to the aforementioned studies in head and neck squamous cell carcinoma and ovarian cancer, the following studies also prove this point. Study in bladder cancer showed miR-934 down-regulated UBE2N protein expression by binding to 3'-UTR of ubiquitin-conjugating enzyme $2 \mathrm{~N}$ (UBE2N) mRNA, thereby inhibiting UBE2N-dependent degradation of CDK6 cyclin and promoting bladder cancer cell growth. ${ }^{7}$ Recent studies have found that there are multiple microRNA expression differences in lung adenocarcinoma patients with EGFR exon 19 deletion compare with wild-type EGFR patients. The expression of miR-934 is up-regulated, especially in triple-negative 

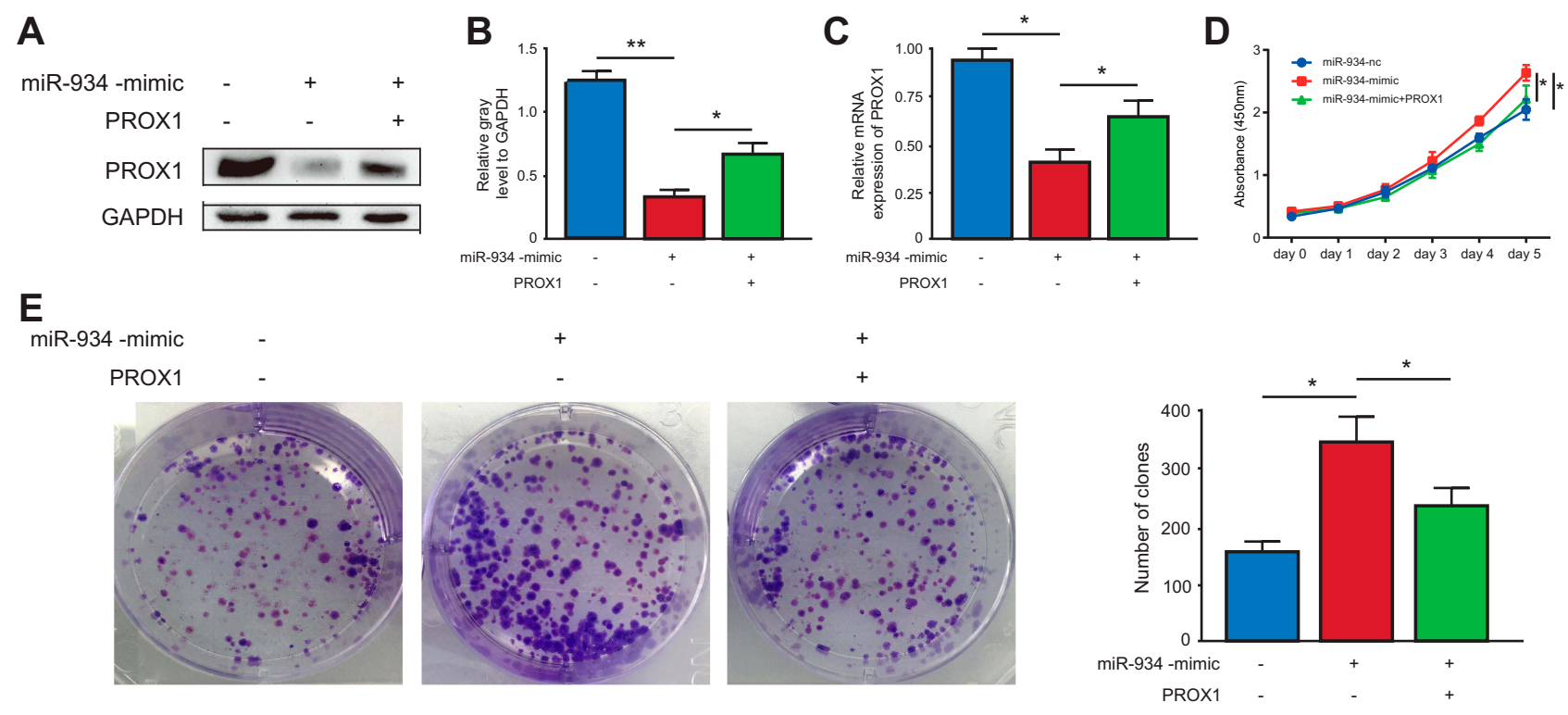

$\mathbf{F}$

miR-934 -mimic

PROX1
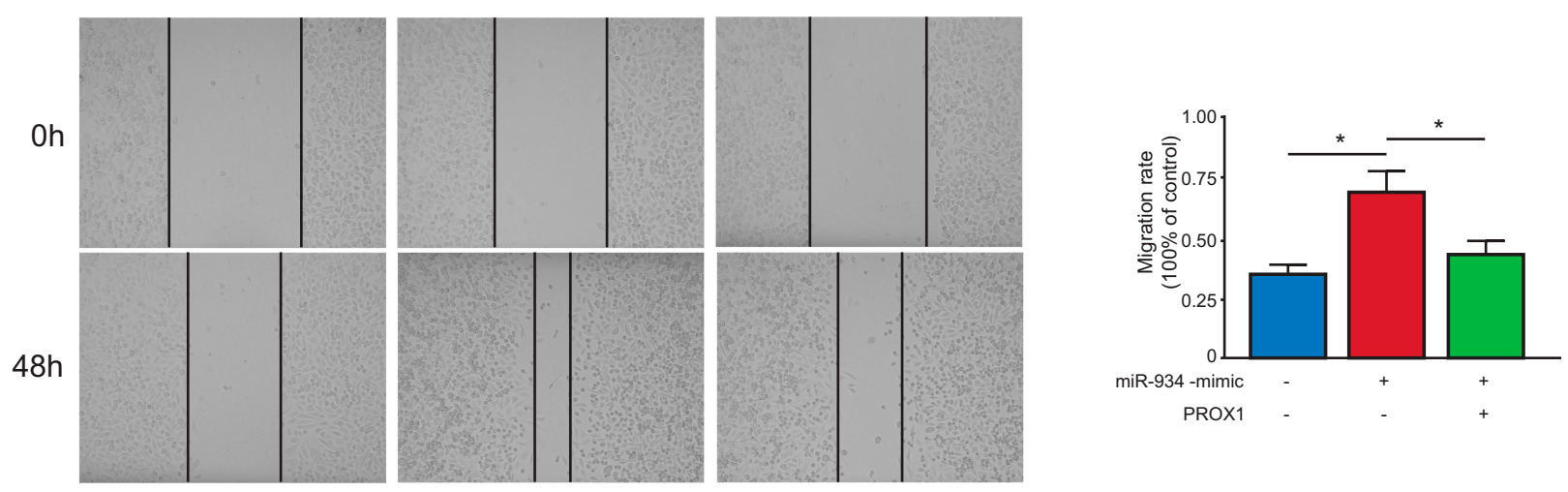

G
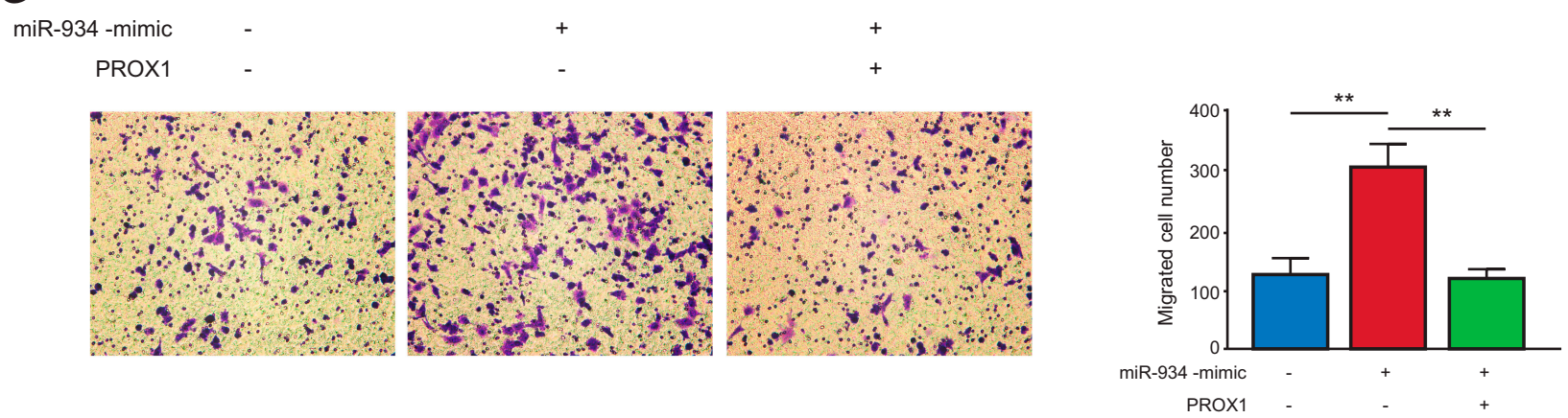

Figure 5 PROXI is necessary for miR-934 to exert its oncogenic function. (A) Western blot used to detect the transfection efficiency in Patu-8988 cells with miR-934-nc, miR-934-mimics and miR-934-mimics+PROXI plasmid. (B) The gray level was quantified by imageJ. (C) Transfection efficiency measured by qRT-PCR. (D) CCK8 assay used to detect cell viability of pancreatic cancer cell in miR-934-nc, miR-934-mimics and miR-934-mimics+PROXI group. (E) Left panel: colony formation assay to detect cell proliferation capacity. Right panel: the colony number was quantified using imagej. (F, $\mathbf{G})$ Left panel: wounding healing and transwell assays to determine the migration ability of pancreatic cancer in miR-934-nc, miR-934-mimics and miR-934-mimics+PROXI group. Right panel: the migration rate and migrated cell number were quantified and analyzed by imagej. $* \mathrm{P}<0.05, * * \mathrm{P}<0.01$.

invasive ductal carcinoma, but the mechanism remains to be studied. ${ }^{19}$ Study by Castilla et al showed that the synergistic expression of VGLL1 and miR-934 may affect the luminal progenitor phenotype through partly regulation of ESR $1 .{ }^{20} \mathrm{In}$ this study, our results indicate that miR-934 is upregulated in pancreatic cancer tissue compared with normal tissue and 
multiple cell lines of pancreatic cancer. Furthermore, it was confirmed by in vitro assays that miR-934 was positively correlated with pancreatic cancer cell proliferation, and migration. Combined with the TCGA database and clinical samples, miR-934 overexpression is also found to be a potential biomarker of poor prognosis in patients.

The PROX1 gene plays an important role in embryonic development in normal individuals, and its mechanism of action in tumor development and progression has been extensively studied. It is noteworthy that it plays this completely opposite role in different types of tumors, and the mechanisms of cancer suppression and carcinogenesis involved are not fully understood. Among the digestive tract tumors, the current thorough research on PROX1 is in colorectal cancer and liver cancer. Interestingly, the development of PROX1 in both tumors seems to play a completely opposite role. ${ }^{21}$ PROX1 has been identified as a downstream target of the APC/ $\beta$-catenin/TCF pathway in colorectal cancer. Through the aberrant activation of this pathway, PROX1 is often up-regulated, which promotes the progression of colorectal cancer. ${ }^{22}$ In HCC cells, the expression of Prox 1 is not regulated by $\beta$ catenin, and clinical data indicate that high expression of Prox 1 is associated with a good prognosis in patients. ${ }^{23}$ In addition, studies have shown that Prox 1 can inhibit the development of HCC by down-regulating Twist expression and decreasing promoter activity to induce a p53dependent senescence-like phenotype. ${ }^{24}$ Saukkonen et al showed that the $\beta$-catenin-PROX1 pathway was inhibited in pancreatic cancer, while the expression of high $\beta$ catenin predicted a better prognosis. ${ }^{14}$ Our study supports the conclusion that PROX1 plays a tumor suppressor role in pancreatic cancer, and the expression of miR-934 is negatively correlated with PROX1. The role of miR-934 and PROX1 in pancreatic cancer still needs to be further studied and understanding its related mechanisms may provide us more options for designing new antipancreatic cancer therapies.

\section{Conclusion}

In summary, our study demonstrated that miR-934 could serve as a prognostic marker and promoted cell growth and metastasis of pancreatic cancer by inhibiting the expression of PROX1. To our knowledge, this is the first report to link miR-934 and PROX1 to explain the mechanism of pancreatic cancer development. Our study leads to the possibility of application of miR-934 and PROX1 in the clinical work of pancreatic cancer.

\section{Acknowledgments}

This study was supported by grants from Shanghai Anticancer Association (EYAS PROJECT; no. SACACY1C19) and Ruijin Youth NSFC Cultivation Fund.

\section{Disclosure}

The authors declare that there are no conflicts of interest.

\section{References}

1. John PN, Jörg K, Patrick M, et al. Therapeutic developments in pancreatic cancer: current and future perspectives. Nat Rev Gastroenterol Hepatol. 2018;15(6):333-348. doi:10.1038/s41575018-0005-x

2. Aatur DS, Eugene JK, Suresh TC, et al. Early detection of pancreatic cancer: opportunities and challenges. Gastroenterology. 2019;156 (7):2024-2040. doi:10.1053/j.gastro.2019.01.259

3. Li Z, Rana TM. Therapeutic targeting of microRNAs: current status and future challenges. Nat Rev Drug Discov. 2014;13(8):622-638. doi:10.1038/nrd4359

4. Hayes J, Peruzzi PP, Lawler S. MicroRNAs in cancer: biomarkers, functions and therapy. Trends Mol Med. 2014;20(8):460-469. doi:10.1016/j.molmed.2014.06.005

5. Maarouf AS, Selena ZK, Elham R, et al. Alcohol-dysregulated miR-30a and miR-934 in head and neck squamous cell carcinoma. Mol Cancer. 2015;14(1):181. doi:10.1186/s12943-015-0452-8

6. Hu Y, Zhang Q, Cui J, et al. Oncogene miR-934 promotes ovarian cancer cell proliferation and inhibits cell apoptosis through targeting BRMS1L. Eur Rev Med Pharmacol Sci. 2019;23(13):5595-5602. doi:10.26355/eurrev_201907_18293

7. Yan H, Ren S, Lin Q, et al. Inhibition of UBE2N-dependent CDK6 protein degradation by mir-934 promotes human bladder cancer cell growth. FASEB J. 2019;33(11):12112-12123. doi:10.1096/ fj.201900499RR

8. Nicole CJ, Miriam ED, Peter B, et al. Lymphatic endothelial cell identity is reversible and its maintenance requires Prox1 activity. Genes Dev. 2008;22(23):3282-3291. doi:10.1101/gad.1727208

9. Jenny H, Sarika H, Pauliina K, et al. Transcription factor PROX1 suppresses notch pathway activation via the nucleosome remodeling and deacetylase complex in colorectal cancer stem-like cells. Cancer Res. 2018;78(20):5820-5832. doi:10.1158/0008-5472.CAN-18-0451

10. Zhang B, Ji S, Ma F, Ma Q, Lu X, Chen X. miR-489 acts as a tumor suppressor in human gastric cancer by targeting PROX1. Am J Cancer Res. 2016;6(9):2021-2030.

11. Choi D, Ramu S, Park E, et al. Aberrant activation of notch signaling inhibits PROX1 activity to enhance the malignant behavior of thyroid cancer cells. Cancer Res. 2016;76(3):582-593. doi:10.1158/00085472.CAN-15-1199

12. Drosos Y, Neale G, Ye J, et al. Prox1-heterozygosis sensitizes the pancreas to oncogenic Kras-induced neoplastic transformation. Neoplasia. 2016;18(3):172-184. doi:10.1016/j.neo.2016.02.002

13. Wang J, Kilic G, Aydin M, et al. Prox1 activity controls pancreas morphogenesis and participates in the production of "secondary transition" pancreatic endocrine cells. Dev Biol. 2005;286(1):182-194. doi:10.1016/j.ydbio.2005.07.021

14. Saukkonen K, Hagström J, Mustonen H, et al. PROX1 and beta-catenin are prognostic markers in pancreatic ductal adenocarcinoma. BMC Cancer. 2016;16(1):472. doi:10.1186/ s12885-016-2497-5

15. Satoh K, Hamada S, Shimosegawa T. Involvement of epithelial to mesenchymal transition in the development of pancreatic ductal adenocarcinoma. J Gastroenterol. 2015;50(2):140-146. doi:10.1007/ s00535-014-0997-0 
16. Gregory PA, Bert AG, Paterson EL, et al. The miR-200 family and miR-205 regulate epithelial to mesenchymal transition by targeting ZEB1 and SIP1. Nat Cell Biol. 2008;10(5):593-601. doi:10.1038/ ncb1722

17. Rawat M, Kadian K, Gupta Y, et al. MicroRNA in pancreatic cancer: from biology to therapeutic potential. Genes (Basel). 2019;10(10). doi:10.3390/genes10100752

18. Ganepola GA, Rutledge JR, Suman P, et al. Novel blood-based microRNA biomarker panel for early diagnosis of pancreatic cancer. World J Gastrointest Oncol. 2014;6(1):22-33. doi:10.4251/wjgo.v6.11.22

19. Ju L, Han M, Li X, Zhao C. MicroRNA signature of lung adenocarcinoma with EGFR Exon 19 Deletion. $J$ Cancer. 2017;8 (7):1311-1318. doi:10.7150/jca.17817

20. Castilla MA, López-García MA, Atienza MR, et al. VGLL1 expression is associated with a triple-negative basal-like phenotype in breast cancer. Endocr Relat Cancer. 2014;21(4):587-599. doi:10.1530/ ERC-13-0485
21. Elsir T, Smits A, Lindström MS, Nistér M. Transcription factor PROX1: its role in development and cancer. Cancer Metastasis Rev. 2012;31(3-4):793-805. doi:10.1007/s10555-012-9390-8

22. Petrova TV, Nykänen A, Norrmén C, et al. Transcription factor PROX1 induces colon cancer progression by promoting the transition from benign to highly dysplastic phenotype. Cancer Cell. 2008;13 (5):407-419. doi:10.1016/j.ccr.2008.02.020

23. Shimoda M, Takahashi M, Yoshimoto T, et al. A homeobox protein, Prox 1, is involved in the differentiation, proliferation, and prognosis in hepatocellular carcinoma. Clin Cancer Res. 2006;12 (20):6005-6011. doi:10.1158/1078-0432.CCR-06-0712

24. Chang TM, Hung WC. The homeobox transcription factor Prox 1 inhibits proliferation of hepatocellular carcinoma cells by inducing p53-dependent senescence-like phenotype. Cancer Biol Ther. 2013;14(3):222-229. doi:10.4161/cbt.23293

\section{Publish your work in this journal}

OncoTargets and Therapy is an international, peer-reviewed, open access journal focusing on the pathological basis of all cancers, potential targets for therapy and treatment protocols employed to improve the management of cancer patients. The journal also focuses on the impact of management programs and new therapeutic agents and protocols on patient perspectives such as quality of life, adherence and satisfaction. The manuscript management system is completely online and includes a very quick and fair peer-review system, which is all easy to use. Visit http://www.dovepress.com/ testimonials.php to read real quotes from published authors. 